\title{
ENTIRE POSITIVE SOLUTIONS FOR INHOMOGENEOUS SEMILINEAR ELLIPTIC SYSTEMS*
}

\author{
QIUYI DAI \\ Department of Applied Mathematics, Hunan University, Changsha Hunan 410082, P.R. China \\ e-mail:Daiqiuyi@yahoo.com.cn
}

(Received 9 February, 2004; accepted 15 September, 2004)

\begin{abstract}
In this paper we investigate existence and nonexistence of positive solutions for inhomogeneous semilinear elliptic systems in $R^{n}$. A criteria of existence and nonexistence of positive solutions is given by the exponents of the rate of nonlinear terms at infinity.
\end{abstract}

2000 Mathematics Subject Classification. 35J60, 35J45.

1. Introduction. The main objective of this paper is to study the existence and nonexistence problems for inhomogeneous semilinear elliptic systems

$$
\begin{cases}-\triangle u=v^{p}+f(x), & x \in R^{n}, \\ -\Delta v=u^{q}+g(x), & x \in R^{n}, \\ u(x)>0, v(x)>0 . & x \in R^{n},\end{cases}
$$

In homogeneous case, i.e. $f=g=0$, problem (1.1) and its extension

$$
\begin{cases}-\Delta u=f(x, v), & x \in \Omega, \\ -\Delta v=g(x, u), & x \in \Omega, \\ u(x)=0, v(x)=0 . & x \in \partial \Omega,\end{cases}
$$

have been extensively studied in bounded domains and $R^{n}$, see [5], [10], [11], [13], [17], [21] etc. Finite energy solutions of the problem (1.2) are usually found as critical points of associated functional. This kind of systems are so-called Hamitonian elliptic system which enjoys some special features. The first one is that the associated functional is strongly indefinite at zero, that is, the quadratic part of the functional is positive definite and negative definite on infinite dimensional subspaces of the Sobolev space where the functional defined on. So there is not anymore mountain pass structure for the associated functional but linking one; the second one is that the natural restriction of $p$ and $q$ is no long as a single equation, that is $1<p, q<\frac{n+2}{n-2}$ for $n \geq 3$. By a Pohozaev type identity for systems [19], [23], it is natural to find solutions for $p, q$ below critical hyperbolic, i.e. $p, q$ satisfy

$$
\frac{1}{p+1}+\frac{1}{q+1}>\frac{n-2}{n} .
$$

*This work is supported by NNSF of China (Grant: No.10171029). 
In [11] and [17], existence results were obtained for problem (1.1) by variational arguments. They used suitable inter-and extropolation spaces to replace $H_{0}^{1}(\Omega) \times$ $H_{0}^{1}(\Omega)$, and extend the variational formulation of (1.2) in a Hilbert space setting to the parameter range defined by (1.3). The main idea is to destroy the symmetry between $u$ and $v$ by demanding more regularity of $u$ than of $v$ if $p$ is large and $q$ is small, and vice verse. Thus fractional Sobolev spaces are involved. The work of [11] and [17] has been extended to $R^{n}$ by [14], [21] etc for problem with prototype

$$
\begin{cases}-\triangle u+u=v^{p}, & x \in R^{n}, \\ -\Delta v+v=u^{q}, & x \in R^{n}, \\ u(x)>0, v(x)>0 . & x \in R^{n},\end{cases}
$$

However, one couldn't expect the problem

$$
\begin{cases}-\triangle u=v^{p}, & x \in R^{n}, \\ -\Delta v=u^{q}, & x \in R^{n}, \\ u(x)>0, v(x)>0 . & x \in R^{n}\end{cases}
$$

possesses any solution for $p, q$ satisfying (1.3). In fact, a Liouville type theorem for (1.5) was obtained by [12] with $p, q$ satisfying $1<p, q<\frac{n+2}{n-2}$. It remains open for the rest case.

The situation becomes different when inhomogeneous terms $f, g$ are involved. The critical hyperbolic (1.3) is no long criteria of the range of $p, q$ for existence and nonexistence of problem (1.1). Our first result states nonexistence for problem (1.1).

Suppose $f, g \in C_{l o c}^{0, \alpha}\left(R^{n}\right)$ are nonnegative and we denote throughout this paper that $\alpha=\frac{1+p}{p q-1}, \beta=\frac{1+q}{p q-1}$.

THEOREM 1.1. If $\max \{\alpha, \beta\} \geq \frac{n-2}{2}$, then there is no solution of problem (1.1).

In the case $n \geq 3$, Theorem 1.1 is proved in [24] via a nonexistence result of Cauchy problem of semilinear parabolic systems. However, the argument is not valid for $n=1,2$, since in that argument one needs that the fundamental solution of Laplace operator decay to zero at infinity and this is not the case for $n=1,2$. The cases $n \leq 2$ and $\max \{\alpha, \beta\}<\frac{n-2}{2}$ are more delicate. In these cases, to obtain nonexistence results for problem (1.1), it is usually necessary to know a suitable decaying law of solutions at infinity. A decaying law for a single equation was obtained in [3]. The fundamental tool used there is a result due to [20], see Lemma 2.1 below. Although similar results of [20] are not available for systems, fortunately, we find a way to apply the result to the spherical mean of solutions to problem (1.1), then we show nonexistence by contradiction. Our second result is as follows.

For a function $f$, we denote by $\bar{f}$ the spherical mean of it which is given in Section 2 . Set

$$
C_{p q}=\frac{q-1}{q}\left[\frac{(2 \alpha(n-2-2 \alpha))^{q}}{q}\right]^{\frac{1}{q-1}}+\frac{p-1}{p}\left[\frac{(2 \beta(n-2-2 \beta))^{p}}{p}\right]^{\frac{1}{p-1}} .
$$

TheOREM 1.2. Suppose $p, q>1$ and $\max \{\alpha, \beta\}<\frac{n-2}{2}$. Then problem (1.1) has no solution if either

$$
\int_{1}^{+\infty}\left[r^{2 p \beta} \bar{f}-C_{p q}\right] \frac{d r}{r}=+\infty \quad \text { or } \quad \int_{1}^{+\infty}\left[r^{2 q \alpha} \bar{g}-C_{p q}\right] \frac{d r}{r}=+\infty .
$$


In particular, if $f(x) \geq \frac{A_{1}}{\left(1+|x|^{2}\right)^{p \beta}}$ with $A_{1}>C_{p q}$ or $g(x) \geq \frac{A_{2}}{\left(1+|x|^{2}\right)^{q \alpha}}$ with $A_{2}>C_{p q}$, then problem (1.1) has no solution.

Next we deal with existence problem for problem (1.1). It is known that there are many results in this direction for one equation; for instance [1-4], [6-9], [12-15], [1819], [21], [22-25] and references therein. Most of these works are done by variational method. However, it is not easy to find a suitable space to handle problem (1.1) and apply variational arguments. A loss of compactness also becomes a problem. We shall use sub-super solution arguments to investigate existence for problem (1.1). We have the following result.

THEOREM 1.3. If $p, q>1, \max \{\alpha, \beta\}<\frac{n-2}{2}$, and $f, g \in C_{l o c}^{0, \gamma}\left(R^{n}\right)$ satisfy

$$
f(x) \leq \frac{B_{1}}{\left(1+|x|^{2}\right)^{p \beta}}, \quad g(x) \leq \frac{B_{2}}{\left(1+|x|^{2}\right)^{q \alpha}},
$$

for $B_{1}, B_{2}>0$ small enough, then there is at least one solution $(u, v)$ to problem $(1.1)$.

Theorems 1.2 and 1.3 indicate that the decay exponents imposed on $f(x)$ and $g(x)$ are sharp for existence and nonexistence results. Theorems 1.1 and 1.3 also indicate that $\max \{\alpha, \beta\}=\frac{n-2}{2}$ is the dividing curve in terms of exponents $p$ and $q$ for existence and nonexistence results for problem (1.1).

As a special case, we shall consider the biharmonic problem:

$$
\begin{cases}\triangle^{2} u=u^{p}+f(x), & x \in R^{n}, \\ u(x)>0, & x \in R^{n} .\end{cases}
$$

Problem (1.6) was studied in [11], [17] etc in bounded domains and $R^{n}$ for $1<p \leq \frac{n+4}{n-4}$ by variational method. Since we allow the exponent $p$ to be bigger than $\frac{n+4}{n-4}$, that is the supercritical case for biharmonic problem, it is more convenient to use sub-super solution arguments. However, to ensure the sub-super solution arguments works well for problem (1.6), we must reduce problem (1.6) to the following elliptic systems first

$$
\begin{cases}-\Delta u=v, & x \in R^{n}, \\ -\Delta v=u^{p}+f(x), & x \in R^{n}, \\ u(x)>0, v(x)>0, & x \in R^{n} .\end{cases}
$$

Then in a similar way, we obtain

THEOREM 1.4. If $n \leq 4$, or $n \geq 5$ and $1<p \leq \frac{n}{n-4}$, then problem (1.6) has no solution.

Let

$$
a=\frac{4}{p-1}\left(n-2-\frac{4}{p-1}\right), \quad b=\frac{2(p+1)}{p-1}\left[n-2-\frac{2(p+1)}{p-1}\right]
$$

and

$$
C_{p}=|b-1| 4^{\frac{3 p+1}{(p-1)^{2}}}\left(\lambda_{1}\left(1, \frac{1}{2}\right)\right)^{\frac{p+1}{p-1}}+\frac{a^{\frac{p}{p-1}}(p-1)}{p^{\frac{p}{p-1}}} .
$$


THEOREM 1.5. Assume that $n \geq 5, p>\frac{n}{n-4}$. Then problem (1.6) has no solution if

$$
\int_{1}^{+\infty}\left[r^{\frac{4 p}{p-1}} \bar{f}-C_{p}\right] \frac{d r}{r}=+\infty .
$$
In particular, If $f(x) \geq \frac{A}{\left(1+|x|^{2}\right)^{\frac{2 p}{p-1}}}$ for some positive constant $A>C_{p}$, then problem
(1.6) has no solution.

THEOREM 1.6. Let $n \geq 5, p>\frac{n}{n-4}$. If $f(x) \in C_{\text {loc }}^{0, \gamma}\left(R^{n}\right)$ satisfy $f(x) \leq \frac{B}{\left(1+|x|^{2}\right)^{\frac{2 p}{p-1}}}$ for $B>0$ small enough, then problem (1.6) has at least one solution.

In Section 3, we prove results for problem (1.1). Biharmonic problem will be discussed in Section 4.

2. Preliminary results. In this section, we present some preliminary results. We shall use a result from [20] to get decaying laws for the spherical mean of positive solutions of the problems under consideration. Denote by $\lambda_{1}(\Omega)$ the first eigenvalue of $-\triangle$ in $\Omega$ with zero Dirichlet boundary condition, we have

LEMMA 2.1 ([20]). Let $\Omega$ be a bounded smooth domain of $R^{n}$ and $n \geq 1$. There does not exist any function $u \in C^{2}(\Omega) \cap C^{1}(\bar{\Omega})$ such that

$$
\begin{cases}\Delta u+\lambda u \leq 0, & x \in \Omega \\ u(x)>0, & x \in \partial \Omega\end{cases}
$$

whenever $\lambda>\lambda_{1}(\Omega)$.

An iteration process will be used in our arguments. For this purpose, we define for $k>1$

$$
\begin{aligned}
a_{k} & =\sum_{j=0}^{k-1}(p q)^{j}=a_{k-1}+(p q)^{k-1}, \\
b_{k} & =\sum_{j=0}^{k-1} j(p q)^{k-1-j}=\sum_{j=1}^{k-1} j(p q)^{k-1-j}, \\
d_{k} & =2(1+q) b_{k}+a_{k} .
\end{aligned}
$$

LEMMA 2.2. The following formula hold:

(1) $b_{k+1}=b_{k}+a_{k}$,

(2) $d_{k+1}=d_{k}+2(1+q) a_{k}+(p q)^{k}$.

Proof.

(1)

$$
\begin{aligned}
b_{k+1}-b_{k} & =\sum_{j=0}^{k} j(p q)^{k-j}-\sum_{j=0}^{k-1} j(p q)^{k-1-j} \\
& =\sum_{j=0}^{k} j(p q)^{k-j}-\sum_{j=1}^{k}(j-1)(p q)^{k-j} \\
& =\sum_{j=1}^{k}(p q)^{k-j}=\sum_{j=0}^{k-1}(p q)^{j}=a_{k} .
\end{aligned}
$$


(2)

$$
\begin{aligned}
d_{k}+2(1+q) a_{k}+(p q)^{k} & =2(1+q) b_{k}+a_{k}+2(1+q) a_{k}+(p q)^{k} \\
& =2(1+q)\left(b_{k}+a_{k}\right)+a_{k}+(p q)^{k} \\
& =2(1+q) b_{k+1}+a_{k+1}=d_{k+1} .
\end{aligned}
$$

LEMMA 2.3. If $p q>1$, then we have

(1) $\lim _{k \rightarrow+\infty} \frac{a_{k}}{(p q)^{k}}=\frac{1}{p q-1}$,

(2) $\lim _{k \rightarrow+\infty} \frac{b_{k}}{(p q)^{k}}=\frac{1}{(p q-1)^{2}}$,

(3) $\lim _{k \rightarrow+\infty} \frac{d_{k}}{(p q)^{k}}=\frac{p q+2 q+1}{(p q-1)^{2}}$.

Proof. Since $p q>1$, we know that $\left\{(p q)^{k}\right\}_{k=1}^{\infty}$ is an increasing sequence and $(p q)^{k} \rightarrow+\infty$ as $k \rightarrow+\infty$. By Stolz's theorem, we have

(1) $\lim _{k \rightarrow+\infty} \frac{a_{k}}{(p q)^{k}}=\lim _{k \rightarrow+\infty} \frac{a_{k+1}-a_{k}}{(p q)^{k+1}-(p q)^{k}}=\lim _{k \rightarrow+\infty} \frac{(p q)^{k}}{(p q)^{k}(p q-1)}=\frac{1}{p q-1}$,

(2) $\lim _{k \rightarrow+\infty} \frac{b_{k}}{(p q)^{k}}=\lim _{k \rightarrow+\infty} \frac{b_{k+1}-b_{k}}{(p q)^{k+1}-(p q)^{k}}=\lim _{k \rightarrow+\infty} \frac{a_{k}}{(p q)^{k}(p q-1)}=\frac{1}{(p q-1)^{2}}$,

(3) $\lim _{k \rightarrow+\infty} \frac{d_{k}}{(p q)^{k}}=\lim _{k \rightarrow+\infty} \frac{2(1+q) b_{k}+a_{k}}{(p q)^{k}}=\frac{2(1+q)}{(p q-1)^{2}}+\frac{1}{p q-1}=\frac{p q+2 q+1}{(p q-1)^{2}}$.

We denote by $\bar{u}$ the spherical mean of a function $u \in C\left(R^{n}\right)$; i.e.

$$
\begin{array}{ll}
\bar{u}(r)=\frac{1}{\omega_{n} r^{n-1}} \int_{|x|=r} u(x) d S, & \text { for all } r>0 \text { and } n \geq 2, \\
\bar{u}(r)=\frac{u(r)+u(-r)}{2}, & \text { for all } r>0 \text { and } n=1,
\end{array}
$$

where $\omega_{n}$ is the area of unit sphere in $R^{n}$ and $d S$ is the surface measure. We can verify that

$$
\bar{u}(0)=u(0),\left(\frac{d \bar{u}}{d r}\right)(0)=0, \overline{\Delta u}=\triangle \bar{u} .
$$

Let $\lambda_{1}(R, r), R>r \geq 0$, be the first eigenvalue of $-\triangle$ in $\Omega=\left\{x \in R^{n}|r<| x \mid<\right.$ $R$ \} with zero Dirichlet boundary condition. Denote $C_{1}=4^{\frac{p q+2 p+1}{(p q-1)^{2}}}\left(\lambda_{1}\left(1, \frac{1}{2}\right)\right)^{\frac{(1+p)}{p q-1}}, C_{2}=$ $4^{\frac{p q+2 q+1}{(p q-1)^{2}}}\left(\lambda_{1}\left(1, \frac{1}{2}\right)\right)^{\frac{(1+q)}{p q-1}}$.

Proposition 2.1. Let $(u, v)$ be a solution of problem (1.1), then we have for all $R>0$ that

$$
\bar{u}(R) \leq C_{1}\left(\frac{1}{R}\right)^{\frac{2(1+p)}{p q-1}}, \quad \bar{v}(R) \leq C_{2}\left(\frac{1}{R}\right)^{\frac{2(1+q)}{p q-1}}
$$

Proof. Let $(u, v)$ be a solution of problem (1.1). Using Green's formula and the first equation of (1.1) we obtain

$$
\frac{d \bar{u}(r)}{d r}=\frac{1}{n \omega_{n} r^{n-1}} \int_{B_{r}(0)} \Delta u d x=-\frac{1}{n \omega_{n} r^{n-1}} \int_{B_{r}(0)}\left(v^{p}+f\right) d x \leq 0
$$


for $r \geq 0$. In the same way, we also have

$$
\frac{d \bar{v}(r)}{d r} \leq 0, \text { for } r \geq 0
$$

Taking spherical mean on both sides of the equations in problem (1.1), we get

$$
\begin{array}{ll}
\Delta \bar{u}+\overline{v^{p}+f(x)}=0, & \text { for } r \geq 0, \\
\Delta \bar{v}+\overline{u^{q}+g(x)}=0, & \text { for } r \geq 0, \\
\bar{u}(r)>0, \quad \bar{v}(r)>0, & \text { for } r \geq 0 .
\end{array}
$$

An application of Jensen's inequality yields

$$
\begin{aligned}
\Delta \bar{u}+\bar{v}^{p} \leq 0, & \text { for } r \geq 0, \\
\Delta \bar{v}+\bar{u}^{q} \leq 0, & \text { for } r \geq 0, \\
\bar{u}(r)>0, \quad \bar{v}(r)>0, & \text { for } r \geq 0 .
\end{aligned}
$$

At this stage, there are some differences between the cases $n=1$ and $n \geq 2$. Because the case $n=1$ is very simple, we only deal with the case $n \geq 2$ in the sequel.

Since $\bar{u}(r)$ and $\bar{v}(r)$ are positive decreasing functions, for any $R>0$ and $\varepsilon \in[0,1)$, we have

$$
\begin{aligned}
& \Delta \bar{u}+\frac{\bar{v}^{p}(R)}{\bar{u}(\varepsilon R)} \bar{u}(r) \leq 0, \quad \text { for } \varepsilon R \leq r \leq R, \\
& \triangle \bar{v}+\frac{\bar{u}^{q}(R)}{\bar{v}(\varepsilon R)} \bar{v}(r) \leq 0, \quad \text { for } \varepsilon R \leq r \leq R, \\
& \bar{u}(r)>0, \quad \bar{v}(r)>0 .
\end{aligned}
$$

By Lemma 2.1, we conclude that

$$
\begin{cases}\bar{v}^{p}(R) \leq \lambda_{1}(R, \varepsilon R) \bar{u}(\varepsilon R), & \forall R>0 \\ \bar{u}^{q}(R) \leq \lambda_{1}(R, \varepsilon R) \bar{v}(\varepsilon R), & \forall R>0\end{cases}
$$

Since $\lambda_{1}(R, \varepsilon R) \leq \lambda_{1}\left(R, \frac{1}{2} R\right)$ for any $\varepsilon \in\left[0, \frac{1}{2}\right]$, we obtain from (2.9) that

$$
\begin{cases}\bar{v}^{p}(R) \leq \lambda_{1}\left(R, \frac{1}{2} R\right) \bar{u}(\varepsilon R), & \forall R>0 \\ \bar{u}^{q}(R) \leq \lambda_{1}\left(R, \frac{1}{2} R\right) \bar{v}(\varepsilon R), & \forall R>0 \\ 0 \leq \varepsilon \leq \frac{1}{2} & \end{cases}
$$

Let $\varepsilon \rightarrow 0$ in (2.10), we get

$$
\begin{cases}\bar{v}^{p}(R) \leq \lambda_{1}\left(R, \frac{1}{2} R\right) \bar{u}(0), & \forall R>0 \\ \bar{u}^{q}(R) \leq \lambda_{1}\left(R, \frac{1}{2} R\right) \bar{v}(0), & \forall R>0\end{cases}
$$


Notice that $\lambda_{1}\left(R, \frac{1}{2} R\right)=\frac{\lambda_{1}\left(1, \frac{1}{2}\right)}{R^{2}}$. It follows from (2.11) that

$$
\begin{array}{ll}
\bar{v}(R) \leq\left[\frac{\bar{u}(0) \lambda_{1}\left(1, \frac{1}{2}\right)}{R^{2}}\right]^{\frac{1}{p}}, & \forall R>0 . \\
\bar{u}(R) \leq\left[\frac{\bar{v}(0) \lambda_{1}\left(1, \frac{1}{2}\right)}{R^{2}}\right]^{\frac{1}{q}}, & \forall R>0 .
\end{array}
$$

Let $\varepsilon=\frac{1}{2}$ in (2.9), we obtain

$$
\begin{array}{ll}
\bar{v}^{p}(R) \leq \frac{\lambda_{1}\left(1, \frac{1}{2}\right)}{R^{2}} \bar{u}\left(\frac{R}{2}\right), \quad & \forall R>0 . \\
\bar{u}^{q}(R) \leq \frac{\lambda_{1}\left(1, \frac{1}{2}\right)}{R^{2}} \bar{v}\left(\frac{R}{2}\right), & \forall R>0 .
\end{array}
$$

Substituting (2.13) into (2.14), we get

$$
\bar{v}(R) \leq 4^{\frac{1}{p q}}(v(0))^{\frac{1}{p q}}\left(\frac{\lambda_{1}\left(1, \frac{1}{2}\right)}{R^{2}}\right)^{\frac{1+q}{p q}} .
$$

Inserting (2.16) into (2.15), we obtain

$$
\bar{u}(R) \leq 4^{\frac{1+(1+q)}{p q^{2}}}(v(0))^{\frac{1}{p q^{2}}}\left(\frac{\lambda_{1}\left(1, \frac{1}{2}\right)}{R^{2}}\right)^{\frac{1+q+p q}{p q^{2}}} .
$$

Substituting (2.17) into (2.14) again, we deduce that

$$
\bar{v}(R) \leq 4^{\frac{2(1+q)+1+p q}{(p q)^{2}}}(v(0))^{\frac{1}{(p q)^{2}}}\left(\frac{\lambda_{1}\left(1, \frac{1}{2}\right)}{R^{2}}\right)^{\frac{(1+q)(1+p q)}{(p q)^{2}}} .
$$

Using the notations $a_{k}$ and $d_{k}$ introduced in the beginning of this section, we may rewrite (2.18) as

$$
\bar{v}(R) \leq 4^{\frac{d_{2}}{(p q)^{2}}}(v(0))^{\frac{1}{(p q)^{2}}}\left(\frac{\lambda_{1}\left(1, \frac{1}{2}\right)}{R^{2}}\right)^{\frac{(1+q) a_{2}}{(p q)^{2}}} .
$$

By the induction, we see that for any $k \geq 2$ it holds

$$
\bar{v}(R) \leq 4^{\frac{d_{k}}{(p q)^{k}}}(v(0))^{\frac{1}{(p q)^{k}}}\left(\frac{\lambda_{1}\left(1, \frac{1}{2}\right)}{R^{2}}\right)^{\frac{(1+q) a_{k}}{(p q)^{k}}} .
$$

Letting $k \rightarrow+\infty$ in (2.20), we get from Lemma 2.2 that

$$
\bar{v}(R) \leq 4^{\frac{p q+2 q+1}{(p q-1)^{2}}}\left(\frac{\lambda_{1}\left(1, \frac{1}{2}\right)}{R^{2}}\right)^{\frac{(1+q)}{p q-1}} .
$$

The estimate for $\bar{u}(R)$ can be obtained in a similar way. The proof is complete. 
3. Existence and nonexistence results for problem (1.1). In this section, we consider existence and nonexistence of positive solutions for problem (1.1). First we deal with nonexistence problem. In the case $n \geq 3$, Theorem 1.1 is proved by a nonexistence result for parabolic system. The cases of Theorem 1.2 and $n=1,2$ of Theorem 1.1 are more delicate, it will be studied via the spherical mean of solutions; next, the existence will be investigated by sub-super solution arguments.

3.1. Nonexistence. We commence with the proof of Theorem 1.1. It is known from [24], (see also [7]) that the Cauchy problem of semilinear parabolic systems

$$
\begin{cases}\frac{\partial u}{\partial t}-\Delta u=v^{p}+f(x), & \text { in } R^{n} \times(0, T), \\ \frac{\partial v}{\partial t}-\Delta v=u^{q}+g(x), & \text { in } R^{n} \times(0, T), \\ u(x, 0)=u_{0}(x) \geq 0, & \text { in } R^{n}, \\ v(x, 0)=v_{0}(x) \geq 0, & \text { in } R^{n} .\end{cases}
$$

has no global positive solution for any nontrivial nonnegative pair $(f(x), g(x))$ and $\left(u_{0}(x), v_{0}(x)\right)$ if $\max \{\alpha, \beta\} \geq \frac{n-2}{2}$ and $n \geq 3$.

Proof of Theorem 1.1. In the case $n \geq 3$, suppose by contradiction that problem (1.1) has a solution $(u(x), v(x))$ under the assumption $\max \{\alpha, \beta\} \geq \frac{n-2}{2}$, then for any $u_{0}(x) \leq u(x)$ and $v_{0}(x) \leq v(x),(u(x), v(x))$ is a super-solution of problem (3.1). Hence, by sub-super solution arguments, we see that problem (3.1) has a global positive solution. This is a contradiction to the nonexistence result for (3.1). Now, to complete the proof of Theorem 1.1, we have to handle the case $n=1,2$. Suppose by contradiction that problem (1.1) has a solution $(u(x), v(x))$ for $n=1,2$, then it's spherical mean $(\bar{u}(r), \bar{v}(r))$ satisfies

$$
\begin{cases}\Delta \bar{u}+\overline{\left(v^{p}+f\right)}=0, & \text { for } r \geq 0 \\ \Delta \bar{v}+\overline{\left(u^{q}+g\right)}=0, & \text { for } r \geq 0 \\ \bar{u}(r)>0, \quad \bar{v}(r)>0, & \text { for } r \geq 0 \\ (\bar{u}(0), \bar{v}(0))=(0,0), & \\ \left(\frac{d \bar{u}}{d r}(0), \frac{d \bar{v}}{d r}(0)\right)=(0,0) . & \end{cases}
$$

Multiplying both side of the differential equation in the above problem by $r^{n-1}$ and integrating the result equations over $[0, r]$ yields

$$
\left\{\begin{array}{l}
\frac{d \bar{u}}{d r}+\frac{1}{r^{n-1}} \int_{0}^{r} s^{n-1} \overline{\left(v^{p}+f\right)} d s=0 \\
\frac{d \bar{v}}{d r}+\frac{1}{r^{n-1}} \int_{0}^{r} s^{n-1} \overline{\left(u^{q}+g\right)} d s=0 .
\end{array}\right.
$$


For any $2 R>r>0$, By integrating the equations in (3.2) over $[r, 2 R]$, we obtain

$$
\begin{aligned}
& \bar{u}(2 R)-\bar{u}(r)+\int_{r}^{2 R} \frac{1}{t^{n-1}} \int_{0}^{t} s^{n-1} \overline{\left(v^{p}+f\right)}(s) d s d t=0, \\
& \bar{v}(2 R)-\bar{v}(r)+\int_{r}^{2 R} \frac{1}{t^{n-1}} \int_{0}^{t} s^{n-1} \overline{\left(u^{q}+g\right)}(s) d s d t=0 .
\end{aligned}
$$

If the Fubini's theorem is employed, these equations become

$$
\begin{aligned}
& \bar{u}(2 R)-\bar{u}(r)+\int_{0}^{r} \int_{r}^{2 R} \frac{s^{n-1}}{t^{n-1}} \overline{\left(v^{p}+f\right)}(s) d t d s+\int_{r}^{2 R} \int_{s}^{2 R} \overline{s^{n-1}} \overline{t^{n-1}} \overline{\left(v^{p}+f\right)}(s) d t d s=0, \\
& \bar{v}(2 R)-\bar{v}(r)+\int_{0}^{r} \int_{r}^{2 R} \frac{s^{n-1}}{t^{n-1}} \overline{\left(u^{q}+g\right)}(s) d t d s+\int_{r}^{2 R} \int_{s}^{2 R} \overline{s^{n-1}} \overline{t^{n-1}} \overline{\left(u^{q}+g\right)}(s) d t d s=0 .
\end{aligned}
$$

From which we have

$$
\begin{aligned}
& \bar{u}(2 R)-\bar{u}(r)+\int_{0}^{r} \int_{r}^{2 R} \frac{s^{n-1}}{t^{n-1}} \overline{\left(v^{p}+f\right)}(s) d t d s \leq 0, \\
& \bar{v}(2 R)-\bar{v}(r)+\int_{0}^{r} \int_{r}^{2 R} \frac{s^{n-1}}{t^{n-1}} \overline{\left(u^{q}+g\right)}(s) d t d s \leq 0 .
\end{aligned}
$$

Thus, for $n=2$, we have

$$
\begin{aligned}
& \int_{0}^{r} \overline{s\left(v^{p}+f\right)}(\ln (2 R)-\ln r) d s \leq \bar{u}(r)-\bar{u}(2 R), \\
& \int_{0}^{r} s \overline{\left(u^{q}+g\right)}(\ln (2 R)-\ln r) d s \leq \bar{v}(r)-\bar{v}(2 R),
\end{aligned}
$$

and for $n=1$, we have

$$
\begin{aligned}
& \int_{0}^{r} \overline{\left(v^{p}+f\right)}(2 R-r) d s \leq \bar{u}(r)-\bar{u}(2 R), \\
& \int_{0}^{r} \overline{\left(u^{q}+g\right)}(2 R-r) d s \leq \bar{v}(r)-\bar{v}(2 R),
\end{aligned}
$$

Choose $r=R$, we obtain

$$
\begin{aligned}
& \ln 2 \int_{0}^{R} s \overline{s\left(v^{p}+f\right)} d s \leq \bar{u}(R)-\bar{u}(2 R), \quad \text { for } n=2, \\
& \ln 2 \int_{0}^{R} s \overline{\left(u^{q}+g\right)} d s \leq \bar{v}(R)-\bar{v}(2 R), \quad \text { for } n=2,
\end{aligned}
$$

and

$$
\begin{array}{ll}
\int_{0}^{R} \overline{\left(v^{p}+f\right)} d s \leq \frac{\bar{u}(R)-\bar{u}(2 R)}{R}, & \text { for } n=1, \\
\int_{0}^{R} \overline{\left(u^{q}+g\right)} d s \leq \frac{\bar{v}(R)-\bar{v}(2 R)}{R}, \quad \text { for } n=1 .
\end{array}
$$


Letting $R \rightarrow+\infty$, by Proposition 2.1 we may conclude that $(u(x), v(x)) \equiv 0$ in both cases $n=2$ and $n=1$. A contradiction.

Now we turn to the case $p, q>1, \max \{\alpha, \beta\}<\frac{n-2}{2}$ and $n \geq 3$.

Proof of Theorem 1.2. Suppose that $(u(x), v(x))$ is a positive solution of problem (1.1), then by the Jensen's inequality we have

$$
\begin{cases}\triangle \bar{u}+\bar{v}^{p}+\bar{f} \leq 0, & \text { for } r \geq 0 \\ \triangle \bar{v}+\bar{u}^{q}+\bar{g} \leq 0, & \text { for } r \geq 0 \\ \bar{u}(r)>0, \quad \bar{v}(r)>0, & \text { for } r \geq 0\end{cases}
$$

Let

$$
\left\{\begin{array}{l}
H(t)=r^{2 \alpha} \bar{u}(r)=r^{\frac{2(1+p)}{p q-1}} \bar{u}(r) \\
h(t)=r^{2 \beta} \bar{v}(r)=r^{\frac{2(1+q)}{p q-1}} \bar{v}(r) \\
t=\log r
\end{array}\right.
$$

It follows from Proposition 2.1 that $r^{2 \alpha} \bar{u}$ and $r^{2 \beta} \bar{v}$ are bounded for $r \geq 1$. Moreover, we may verify that $H(t)$ and $h(t)$ satisfy

$$
\begin{gathered}
H^{\prime \prime}(t)+d_{1} H^{\prime}(t)-a H(t)+h^{p}(t)+e^{2(\alpha+1)} \bar{f} \leq 0, \quad \text { for } t>0, \\
h^{\prime \prime}(t)+d_{2} h^{\prime}(t)-b h(t)+H^{q}(t)+e^{2(\beta+1)} \bar{g} \leq 0, \quad \text { for } t>0
\end{gathered}
$$

where

$$
\begin{gathered}
d_{1}=n-2-4 \alpha, \quad d_{2}=n-2-4 \beta, \\
a=2 \alpha(n-2-2 \alpha), \quad b=2 \beta(n-2-2 \beta) .
\end{gathered}
$$

Set

$$
\left\{\begin{array}{l}
G(H, h)=a H-H^{q}+b h-h^{p} \\
F(\bar{f}, \bar{g})=e^{2(\alpha+1)} \bar{f}+e^{2(\beta+1)} \bar{g}
\end{array}\right.
$$

Then, by (4.1) and (4.2), we have

$$
(H+h)^{\prime \prime}(t)+d_{1} H^{\prime}(t)+d_{2} h^{\prime}(t)-G(H(t), h(t))+F(\bar{f}, \bar{g}) \leq 0, \quad \text { for } t>0 .
$$

It is easy to check that

$$
\max G(H, h)=C_{p q}
$$

Therefore

$$
(H+h)^{\prime \prime}(t)+d_{1} H^{\prime}(t)+d_{2} h^{\prime}(t)-C_{p q}+F(\bar{f}, \bar{g}) \leq 0, \text { for } t>0 .
$$

Integrating the above inequality over $[0, T]$, we obtain

$$
(H+h)^{\prime}(T) \leq(H+h)^{\prime}(0)-d_{1}(H(T)-H(0))-d_{2}(h(T)-h(0))-\int_{0}^{T}\left(F(\bar{f}, \bar{g})-C_{p q}\right) d \tau
$$


Since $H(t)$ and $h(t)$ are bounded for $t \geq 0$, and by our assumptions

$$
\int_{0}^{T}\left(F(\bar{f}, \bar{g})-C_{p q}\right) d \tau=\int_{1}^{e^{T}}\left(r^{2 p \beta} \bar{f}+r^{2 q \alpha} \bar{g}-C\right) \frac{d r}{r}=+\infty, \quad \text { as } T \rightarrow+\infty,
$$

it follows that

$$
(H+h)^{\prime}(T) \leq-C_{0}<0, \text { for large } T>0,
$$

which in turn implies that

$$
H(T)+h(T)=0, \text { at some } T>0 .
$$

This is a contradiction since $H(t), h(t)>0$ for all $t>0$. The assertion follows.

\subsection{Existence.}

Proof of Theorem 1.3. Theorem 1.3 will be proved by sub-super solution method. It is obvious that $(0,0)$ is a subsolution of problem $(1.1)$. To complete the proof, we look for a supersolution of problem (1.1) of the following type

$$
\left(w_{1}(r), w_{2}(r)\right)=\left(\frac{\xi_{1}}{\left(1+r^{2}\right)^{\alpha_{1}}}, \frac{\xi_{2}}{\left(1+r^{2}\right)^{\alpha_{2}}}\right), \quad r=|x|,
$$

where $\alpha_{1}>0, \alpha_{2}>0, \xi_{1}>0$ and $\xi_{2}>0$ will be specified later. Since

$$
-\triangle w_{i}(r)=-\frac{d^{2} w_{i}}{d r^{2}}-\frac{n-1}{r} \frac{d w_{i}}{d r}, \quad i=1,2,
$$

we deduce

$$
\left\{\begin{array}{l}
-\triangle w_{1}-w_{2}^{p}=-\frac{4 r^{2} \alpha_{1}\left(\alpha_{1}+1\right) \xi_{1}}{\left(1+r^{2}\right)^{\alpha_{1}+2}}+\frac{2 \alpha_{1} \xi_{1} n}{\left(1+r^{2}\right)^{\alpha_{1}+1}}-\frac{\xi_{2}^{p}}{\left(1+r^{2}\right)^{\alpha_{2} p}} \\
-\triangle w_{2}-w_{1}^{q}=-\frac{4 r^{2} \alpha_{2}\left(\alpha_{2}+1\right) \xi_{2}}{\left(1+r^{2}\right)^{\alpha_{2}+2}}+\frac{2 \alpha_{2} \xi_{2} n}{\left(1+r^{2}\right)^{\alpha_{2}+1}}-\frac{\xi_{1}^{q}}{\left(1+r^{2}\right)^{\alpha_{1} q}} .
\end{array}\right.
$$

Choose $\alpha_{1}, \alpha_{2}$ so that

$$
\alpha_{1}+1=\alpha_{2} p, \quad \alpha_{2}+1=\alpha_{1} q
$$

i.e.

$$
\alpha_{1}=\frac{1+p}{p q-1}=\alpha, \quad \alpha_{2}=\frac{1+q}{p q-1}=\beta
$$

Then we have

$$
\left\{\begin{array}{l}
-\triangle w_{1}-w_{2}^{p} \geq \frac{2 \alpha \xi_{1}(n-2-2 \alpha)-\xi_{2}^{p}}{\left(1+r^{2}\right)^{p \beta}} \\
-\triangle w_{2}-w_{1}^{q} \geq \frac{2 \beta \xi_{2}(n-2-2 \beta)-\xi_{1}^{q}}{\left(1+r^{2}\right)^{q \alpha}}
\end{array}\right.
$$


Since $\max \{\alpha, \beta\}<\frac{n-2}{2}$ and $p, q>1$, it follows that

$$
n-2-2 \alpha>0 \text { and } n-2-2 \beta>0 .
$$

Choose $\xi_{1}=\xi_{2}=\xi_{0}>0$ so that

$$
\xi_{0}<\min \left\{(2 \alpha(n-2-2 \alpha))^{\frac{1}{p-1}},(2 \beta(n-2-2 \beta))^{\frac{1}{q-1}}\right\} .
$$

Then, we have

$$
2 \alpha(n-2-2 \alpha) \xi_{0}-\xi_{0}^{p}>0, \quad 2 \beta(n-2-2 \beta) \xi_{0}-\xi_{0}^{q}>0 .
$$

If the positive constants $B_{1}$ and $B_{2}$ are so small that

$$
B_{1} \leq 2 \alpha(n-2-2 \alpha) \xi_{0}-\xi_{0}^{p}, \quad B_{2} \leq 2 \beta(n-2-2 \beta) \xi_{0}-\xi_{0}^{q}
$$

then, by (3.1), we have

$$
\left\{\begin{array}{l}
-\Delta w_{1}-w_{2}^{p} \geq \frac{B_{1}}{\left(1+r^{2}\right)^{p \beta}} \geq f(x), \\
-\Delta w_{2}-w_{1}^{q} \geq \frac{B_{2}}{\left(1+r^{2}\right)^{q \alpha}} \geq g(x) .
\end{array}\right.
$$

This means that $\left(w_{1}(r), w_{2}(r)\right)$ is a supersolution of problem (1.1). By sub-super solution method, we know that problem (1.1) has at least one solution provided that $B_{1}>0$ and $B_{2}>0$ small enough. This completes the proof.

4. Biharmonic equations. To consider the existence and nonexistence for problem (1.6), we reduce it to problem (1.7). We need to show both problems are equivalent. This is proved in Lemma 4.1 .

LEMMA 4.1. A function $u$ is a solution of (1.6) if and only if $(u,-\Delta u)$ is a solution of (1.7).

Proof. Obviously, if $(u, v)$ is a solution of (1.7), then $u$ is a solution of (1.6).

Suppose now $u$ is a solution of (1.6). Let $v=-\Delta u$. It suffices to show that $v(x)>0$ for all $x \in R^{n}$, since then $(u, v)$ is a solution of (1.7). Because $-\Delta u(x)>0$ follows from $-\Delta u(x) \geq 0$ and the strong maximum principle, we have only to prove that $-\Delta u(x) \geq 0$ for all $x \in R^{n}$. By problem (1.6), we know that $u(x)$ and $v(x)$ satisfy

$$
\begin{cases}-\Delta u=v, & x \in R^{n}, \\ -\Delta v=u^{p}+f(x), & x \in R^{n}, \\ u(x)>0, & x \in R^{n} .\end{cases}
$$

Now, we try to prove that $v(x) \geq 0$ for any $x \in R^{n}$. Assume by contradiction that there exists a point $x_{0} \in R^{n}$ such that $v\left(x_{0}\right)<0$. Then we shall try to get a contradiction. Without loss of generality, we may assume that $x_{0}=0$. 
Taking spherical mean on both side of equation in problem (4.1), we get

$$
\begin{cases}-\Delta \bar{u}=\bar{v}, & r>0, \\ -\Delta \bar{v}=\overline{u^{p}+f(x),} & r>0, \\ \bar{u}(r)>0, & r>0 .\end{cases}
$$

Since $\overline{f(x)} \geq 0$ and $p>1$, by Jensen's inequality and (4.2), we have

$$
\begin{array}{ll}
\triangle \bar{u}+\bar{v}=0, & r>0, \\
\triangle \bar{v}+\bar{u}^{p} \leq 0, & r>0 .
\end{array}
$$

Noticing that $\bar{u}(r)>0$ for $r>0$, by (4.4), we have

$$
\triangle \bar{v}=\frac{d^{2} \bar{v}}{d r^{2}}+\frac{n-1}{r} \frac{d \bar{v}}{d r} \leq 0, \text { for all } r>0 .
$$

From which we may deduce that

$$
\frac{d \bar{v}}{d r} \leq 0, \quad \text { for all } r>0 .
$$

This in turn implies that

$$
\bar{v}(r) \leq \bar{v}(0)=v(0)<0, \quad \text { for all } r>0 .
$$

Let $\xi_{0}=-\frac{v(0)}{2 n}>0$. we may derive from (4.3) and (4.5) that

$$
\bar{u}(r) \geq \xi_{0} r^{2}, \quad \text { for all } r>0 .
$$

Next, by mathematical induction method, we claim that for any integer $k \geq 0$ we have

$$
\bar{u}(r) \geq\left[\xi_{0}^{p^{k}} /(n+2 p+2)^{\frac{4\left(p^{k+1}-(k+1) p+k\right)}{(p-1)^{2}}}\right] r^{\frac{2 p^{k+1}+2 p^{k}-4}{p-1}} .
$$

In fact, for the first step of induction method, (4.6) implies that (4.7) hold for $k=0$. Then we assume that (4.7) hold for $k=k$ and try to prove that (4.7) hold also for $k=k+1$. To do this, replacing $\bar{u}$ in (4.4) by (4.7), then multiplying both side of the resulting inequality by $r^{n-1}$ and integrating it twice, we get

$$
\bar{v}(r) \leq-\frac{\xi_{0}^{p^{k+1}}}{\left(n+p a_{k}\right)\left(p a_{k}+2\right)(n+2 p+2)^{p b_{k}}} r^{p a_{k}+2},
$$

where

$$
a_{k}=\frac{2 p^{k+1}+2 p^{k}-4}{p-1}, \quad b_{k}=\frac{4\left(p^{k+1}-(k+1) p+k\right)}{(p-1)^{2}} .
$$

Now, replacing $\bar{v}(r)$ in (4.3) by (4.8), then multiplying both side of the resulting inequality by $r^{n-1}$ and integrating it twice, we may obtain

$$
\bar{u}(r) \geq \frac{\xi_{0}^{p^{k+1}}}{\left(n+p a_{k}\right)\left(p a_{k}+2\right)\left(n+p a_{k}+2\right)\left(p a_{k}+4\right)(n+2 p+2)^{p b_{k}}} r^{p a_{k}+4},
$$


It is easy to see that

$$
p a_{k}+4=\frac{2 p^{k+2}+2 p^{k+1}-4 p}{p-1}+4=\frac{2 p^{k+2}+2 p^{k+1}-4}{p-1}
$$

In addition, by mathematical induction method, we can prove that

$$
\left(n+p a_{k}\right)\left(p a_{k}+2\right)\left(n+p a_{k}+2\right)\left(p a_{k}+4\right) \leq(n+2 p+2)^{4(k+1)} .
$$

This implies that

$$
\begin{aligned}
(n & \left.+p a_{k}\right)\left(p a_{k}+2\right)\left(n+p a_{k}+2\right)\left(p a_{k}+4\right)(n+2 p+2)^{p b_{k}} \\
& \leq(n+2 p+2)^{4(k+1)}(n+2 p+2)^{p b_{k}} \\
& =(n+2 p+2)^{p b_{k}+4(k+1)} \\
& =(n+2 p+2)^{\frac{4\left(p^{k+2}-(k+1) p^{2}+k p\right)}{(p-1)^{2}}+4(k+1)} \\
& =(n+2 p+2)^{\frac{4\left(p^{k+2}-(k+2) p^{2}+k+1\right)}{(p-1)^{2}}}
\end{aligned}
$$

By (4.10), (4.11) and (4.12), we have

$$
\bar{u}(r) \geq\left[\xi_{0}^{p^{k+1}} /(n+2 p+2)^{\frac{4\left(p^{k+2}-(k+2) p+k+1\right)}{(p-1)^{2}}}\right] r^{\frac{2 p^{k+2}+2 p^{k+1}-4}{p-1}}
$$

This implies that (4.7) is valid for $k=k+1$. Hence, the claim is proved.

Now, we continue our proof by proceeding it into the following two cases.

Case 1. If $\xi_{0} \geq 1$, then we choose $r_{0}=(n+2 p+2)^{\frac{4}{p-1}}$. By (4.7), we have

$$
\bar{u}\left(r_{0}\right) \geq \xi_{0}^{p^{k}}(n+2 p+2)^{4 p^{k+1}+8 p^{k}+4(k+1) p-4 k-16}
$$

This is obviously a contradiction, since on the left hand side, $\bar{u}\left(r_{0}\right)$ is a constant independent of $k$, while the right hand side tends to $+\infty$ as $k$ tends to $+\infty$ for $p>1$.

Case 2. If $\xi_{0}<1$, then we choose $r_{0}=\xi_{0}^{-1}(n+2 p+2)^{\frac{4}{p-1}}$. By (4.7), we have

$$
\bar{u}\left(r_{0}\right) \geq\left(\xi_{0}^{-1}\right)^{p^{k+1}+3 p^{k}-4}(n+2 p+2)^{4 p^{k+1}+8 p^{k}+4(k+1) p-4 k-16} .
$$

This is also a contradiction by the same reason as that in Case 1 .

To sum up, we have proved that $-\Delta u \geq 0$ for all $x \in R^{n}$. Finally, applying strong maximum principle to problem (4.1), we conclude that $-\Delta u>0$ for all $x \in R^{n}$. This completes the proof of equivalence between problem (1.6) and (1.7).

By Lemma 4.1, we need only to consider the problem (1.7). In this case $\alpha=\frac{2}{p-1}$ and $\beta=\frac{1+p}{p-1}$. Hence, $\max \{\alpha, \beta\}=\frac{1+p}{p-1}$ since $p>1$. It is obvious that $\frac{1+p}{p-1} \geq \frac{n-2}{2}$ for $n \leq 4$, or $n \geq 5$ and $1<p \leq \frac{n}{n-4}$. So Theorem 1.4 immediately follows from Theorem 1.1. 
Proof of Theorem 1.5. The proof is similar to that of Theorem 1.2, we sketch it.

Suppose by contradiction that $(u(x), v(x))$ is a positive solution of problem (1.7), we would have

$$
\begin{cases}\Delta \bar{u}+\bar{v}=0, & \text { for } r \geq 0 \\ \Delta \bar{v}+\bar{u}^{p}+\bar{f} \leq 0, & \text { for } r \geq 0 \\ \bar{u}(r)>0, \quad \bar{v}(r)>0, & \text { for } r \geq 0\end{cases}
$$

Let

$$
\left\{\begin{array}{l}
H(t)=r^{2 \alpha} \bar{u}(r)=r^{\frac{4}{p-1}} \bar{u}(r), \\
h(t)=r^{2 \beta} \bar{v}(r)=r^{\frac{2(1+p)}{p-1}} \bar{v}(r), \\
t=\log r .
\end{array}\right.
$$

It follows from Proposition 2.1 that $r^{\frac{4}{p-1}} \bar{u} \leq C_{1}$ and $r^{\frac{2(1+p)}{p-1}} \bar{v} \leq C_{2}$ for $r \geq 1$, where $C_{1}=4^{\frac{p+3}{(p-1)^{2}}}\left(\lambda_{1}\left(1, \frac{1}{2}\right)\right)^{\frac{2}{p-1}}$ and $C_{2}=4^{\frac{3 p+1}{(p-1)^{2}}}\left(\lambda_{1}\left(1, \frac{1}{2}\right)\right)^{\frac{1+p}{p-1}}$. Therefore

$$
\begin{aligned}
H^{\prime \prime}(t)+d_{1} H^{\prime}(t)-a H(t)+h(t)=0, & \text { for } t>0, \\
h^{\prime \prime}(t)+d_{2} h^{\prime}(t)-b h(t)+H^{p}(t)+e^{\frac{4 p}{p-1}} \bar{f} \leq 0, & \text { for } t>0,
\end{aligned}
$$

where

$$
\begin{gathered}
d_{1}=n-2-\frac{8}{p-1}, \quad d_{2}=n-2-\frac{4(p+1)}{p-1} \\
a=\frac{4}{p-1}\left(n-2-\frac{4}{p-1}\right), \quad b=\frac{2(p+1)}{p-1}\left(n-2-\frac{2(p+1)}{p-1}\right) .
\end{gathered}
$$

Set

$$
\left\{\begin{array}{l}
G(H, h)=a H-H^{p}+b h-h, \\
F(\bar{f})=e^{\frac{4 p}{p-1}} \bar{f}
\end{array}\right.
$$

Then, by (4.14) and (4.15), we have

$$
(H+h)^{\prime \prime}(t)+d_{1} H^{\prime}(t)+d_{2} h^{\prime}(t)-G(H(t), h(t))+F(\bar{f}) \leq 0, \text { for } t>0 .
$$

It is easy to check that

$$
G(H(t), h(t)) \leq C_{3}=|b-1| C_{2}+\frac{a^{\frac{p}{p-1}}(p-1)}{p^{\frac{p}{p-1}}}, \text { for } t>0 .
$$

Hence

$$
(H+h)^{\prime \prime}(t)+d_{1} H^{\prime}(t)+d_{2} h^{\prime}(t)-C_{3}+F(\bar{f}) \leq 0, \text { for } t>0
$$

which implies

$$
(H+h)^{\prime}(T) \leq(H+h)^{\prime}(0)-d_{1}(H(T)-H(0))-d_{2}(h(T)-h(0))-\int_{0}^{T}\left(F(\bar{f})-C_{3}\right) d \tau .
$$


Since $H(T)$ and $h(T)$ are bounded for $T \geq 0$, and

$$
\int_{0}^{T}\left(F(\bar{f})-C_{3}\right) d \tau=\int_{1}^{e^{T}}\left(r^{\frac{4 p}{p-1}} \bar{f}-C_{3}\right) \frac{d r}{r}=+\infty, \text { as } T \rightarrow+\infty,
$$

it follows that

$$
(H+h)^{\prime}(T) \leq-C_{0}<0, \text { for large } T>0 .
$$

Thus

$$
H(T)+h(T)=0, \text { at some } T>0 .
$$

giving a contradiction.

Proof of Theorem 1.6. We shall use sub-super solution arguments in a similar way as the proof of Theorem 1.3. We sketch the proof.

It is obvious that $(0,0)$ is a subsolution of problem $(1.7)$. Now we look for a supersolution of problem (1.7). Let

$$
\left(w_{1}(r), w_{2}(r)\right)=\left(\frac{\xi_{1}}{\left(1+r^{2}\right)^{\alpha_{1}}}, \frac{\xi_{2}}{\left(1+r^{2}\right)^{\alpha_{2}}}\right), \quad r=|x|,
$$

where $\alpha_{1}>0, \alpha_{2}>0, \xi_{1}>0$ and $\xi_{2}>0$ will be specified later. Therefore

$$
\left\{\begin{array}{l}
-\triangle w_{1}-w_{2}=-\frac{4 r^{2} \alpha_{1}\left(\alpha_{1}+1\right) \xi_{1}}{\left(1+r^{2}\right)^{\alpha_{1}+2}}+\frac{2 \alpha_{1} \xi_{1} n}{\left(1+r^{2}\right)^{\alpha_{1}+1}}-\frac{\xi_{2}}{\left(1+r^{2}\right)^{\alpha_{2}}}, \\
-\triangle w_{2}-w_{1}^{p}=-\frac{4 r^{2} \alpha_{2}\left(\alpha_{2}+1\right) \xi_{2}}{\left(1+r^{2}\right)^{\alpha_{2}+2}}+\frac{2 \alpha_{2} \xi_{2} n}{\left(1+r^{2}\right)^{\alpha_{2}+1}}-\frac{\xi_{1}^{p}}{\left(1+r^{2}\right)^{\alpha_{1} p}} .
\end{array}\right.
$$

Choosing $\alpha_{1}, \alpha_{2}$ so that

$$
\alpha_{1}+1=\alpha_{2}, \quad \alpha_{2}+1=\alpha_{1} p
$$

i.e.

$$
\alpha_{1}=\frac{2}{p-1}=\alpha, \quad \alpha_{2}=\frac{1+p}{p-1}=\beta,
$$

then we obtain

$$
\left\{\begin{array}{l}
-\Delta w_{1}-w_{2} \geq \frac{2 \alpha \xi_{1}(n-2-2 \alpha)-\xi_{2}}{\left(1+r^{2}\right)^{\beta}} \\
-\Delta w_{2}-w_{1}^{p} \geq \frac{2 \beta \xi_{2}(n-2-2 \beta)-\xi_{1}^{p}}{\left(1+r^{2}\right)^{p \alpha}}
\end{array}\right.
$$

Since $n \geq 5$ and $p>\frac{n}{n-4}$, it follows that

$$
n-2-2 \alpha>0 \text { and } n-2-2 \beta>0 .
$$

Choose $\xi_{1}>0$ and $\xi_{2}>0$ so that

$$
\xi_{2}=2 \alpha(n-2-2 \alpha) \xi_{1}, \quad \xi_{1}<[4 \alpha \beta(n-2-2 \alpha)(n-2-2 \beta)]^{\frac{1}{p-1}} .
$$


Then, we have

$$
2 \alpha(n-2-2 \alpha) \xi_{1}-\xi_{2}=0, \quad 2 \beta(n-2-2 \beta) \xi_{2}-\xi_{1}^{p}>0 .
$$

If $B$ in Theorem 1.6 is so small that

$$
B \leq 2 \beta(n-2-2 \beta) \xi_{2}-\xi_{1}^{p}
$$

then,

$$
\left\{\begin{array}{l}
-\Delta w_{1}-w_{2} \geq 0, \\
-\triangle w_{2}-w_{1}^{p} \geq \frac{B}{\left(1+r^{2}\right)^{p \alpha}} \geq f(x) .
\end{array}\right.
$$

This means that $\left(w_{1}(r), w_{2}(r)\right)$ is a supersolution of problem (1.7). By sub-super solution method, we know that problem (1.7) has at least one solution provided $B$ small enough. This completes the proof.

\section{REFERENCES}

1. S. Adachi and K. Tanaka, Four positive solutions for the semilinear elliptic equations $-\triangle u+u=a(x) u^{p}+f(x)$ in $R^{n}$, Calc. Var. PDEs 11 (2000), 63-95.

2. S. Bae and W. M. Ni, Existence and infinite multiplicity for an inhomogeneous semilinear elliptic equation on $R^{n}$, Math. Ann. 320 (2001), 191-210.

3. G. Bernard, An inhomogeneous semilinear equation in entire space, J. Diff. Eqns. 125 (1996), 184-214.

4. D. M. Cao and H. S. Zhou, Multiple positive solutions of nonhomogeneous semilinear elliptic equations in $R^{N}$, Proc. Royal Soc. Edinburgh, Sect. A 126 (1996), 443-463.

5. Ph. Clement, D. G. Figuereido and E. Mitidieri, Positive solutions of semilinear elliptic systems, Comm. P.D.E., 17 (1992), 923-940.

6. Q. Y. Dai and Y. G. Gu, Positive solutions for non-homogeneous semilinear elliptic equations with data that changes sign, Proc. Royal Soc. Edinburgh, Sect. A 133 (2003), to appear.

7. K. Deng and H. A. Levine, The role of critical exponents in blow up theorems, The sequel, J. Math. Anal. Appl. 243 (2000), 85-126.

8. Y. B. Deng, Existence of multiple positive solutions of inhomogeneous semilinear elliptic problem involving critical exponents, Comm. PDEs 17 (1992), 33-53.

9. Y. B. Deng, Y. Li and X. J. Zhao, Multiple solutions and bifurcation of nonhomogeneous semilinear equations in $R^{N}$, in Progress in nonlinear analysis, Nankai Series in Pure and Applied Mathematics and Theoretical Physics, Vol. 6 (ed. K. C. Chang and Y. M. Long) (World Scientific, 1999), 81-102.

10. Yanheng Ding and Shujie Li, Existence of entire solutions for some elliptic systems, Bull. Austral. Math.Soc. 50 (1994), 501-519.

11. D. G. de Figueiredo and P. L. Felmer, On superquadratic elliptic systems, Trans. Amer. Math. Soc. 343 (1994), 99-116.

12. D. G. de Figueiredo and P. L. Felmer, A Liouville type theorem for systems, Ann. Scuola Norm. Pisa XXI (1994), 387-397.

13. D. G. Figueiredo and J. F. Yang, Apriori bounds for positive solutions of a nonvariational elliptic system, Comm. PDEs 26 (2001), 2305-2321.

14. D. G. de Figueiredo and Jianfu Yang, Decay, symmetry and existence of solutions of semilinear elliptic systems, Nonlinear Anal. 33 (1998), 211-234.

15. D. Gilbarg and N. S. Trudinger, Elliptic partial differential equations of second order (Springer-Verlag, 2001).

16. N. Hirano, Existence of entire positive solutions for nonhomogeneous elliptic equations, Nonlinear Anal. 29 (1997), 889-901. 
17. J. Hulshof and R. C. A. M. Van de Vorst, Differential systems with strongly indefinite variational structure, J. Funct. Anal. 114 (1993), 32-58.

18. L. Jeanjean, Two positive solutions for a class of nonhomogeneous elliptic equations, Diff. Integ. Eqns. 10 (1997), 609-624.

19. E. Mitidieri, A Rellich type identity and applications, Comm. PDEs 33 (1998), 211-234.

20. M. Ramaswamy, On the global set of solutions of a nonlinear ODE: theoretical and numerical description, J. Diff. Eqns. 65 (1986), 1-48.

21. B. Sirakov, On the existence of solutions of Hamiltonian elliptic systems in $R^{N}, A d v$. Differential Equations 5 (2000), 1445-1464.

22. G. Tarantello, On nonhomogeneous elliptic equations involving critical Sobolev exponent, Ann. Inst. H. Poincaré Anal. Non Linéaire 9 (1992), 281-304.

23. R. C. A. M. Van de Vorst, Variational identities and applications to differential systems, Arch. Rational Mech. Anal. 116 (1991), 375-398.

24. Q. S. Zhang, Blow-up and global existence of solutions to an inhomogeneous parabolic system, J. Diff. Eqns. 147 (1998), 155-183.

25. X. P. Zhu, A perturbation result on positive entire solutions of a semilinear elliptic equation, J. Diff. Eqns. 92 (1991), 163-178.

26. X. P. Zhu and H. S. Zhou, Existence of multiple positive solutions of inhomogeneous semilinear elliptic problems in unbounded domains, Proc. Royal Soc. Edinburgh Sect. A 115 (1990), 301-318. 